Military Technical College Kobry El-Kobbah, Cairo, Egypt

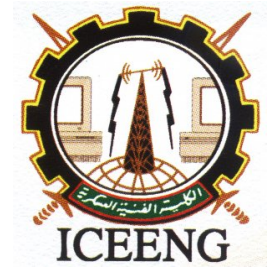

\author{
$7^{\text {th }}$ International Conference \\ on Electrical Engineering \\ ICEENG 2010
}

\title{
Applications of H-infinity loop shaping design procedure in UAV Flight Control Systems
}

\author{
By \\ Elfatih G. Hamdi * \\ Ahmed M. Youssef** \\ Gamal A. Elsheikh ***
}

\section{Abstract:}

The UAV flight control system is rich with attractive and challenging design problems to achieve robust stability and acceptable performance across specified flight envelope in the presence of uncertainties. Therefore, this paper is devoted to design an adequate flight control system for stabilizing a fixed wing (Aerosonde) UAV under exogenous inputs. This UAV is modeled and it is utilized with the $\mathrm{H}_{\infty}$ loop-shaping design procedure (LSDP) to design the necessary flight control system such that the performance requirements are achieved. This work is compared with a previous one using the classical controllers in terms of performance and stability robustness including disturbance rejection, noise attenuation, unmodeled dynamics, and control effort. The obtained results clarify the ability of the designed system using $\mathrm{H}_{\infty}$ to cope with the specified levels of uncertainty including unmodeled dynamics, disturbances and measurement noise. In addition, it reveals its superior capabilities upon the classical techniques.

\section{Keywords:}

UAV, Flight Control, Robust Control Techniques

* Karary University, Khartoum, Sudan

** Military Technical College, Cairo, Egypt

*** Science Valley Academy, Cairo, Egypt 


\section{Introduction:}

The $\mathrm{H}_{\infty}$ loop-shaping design procedure (LSDP) is a sensible and powerful procedure combined with the classical loop shaping of the open loop system frequency response to have a desired loop shape. It is apart of the $\mathrm{H}$-infinity optimization problem that has been developed by McFarlane and Glover. The feature of this technique is that the closed loop requirements (disturbance rejection and noise attenuation properties) can be specified by shaping the open-loop gains. The obtained controller is robust against the normalized coprime factor uncertainty.

Whenever the LSDP is used, the optimal robust controller can be limited to solve two Riccati equations [1]; (Control Algebraic Riccati Equation (CARE) and Filter Algebraic Riccati Equation (FARE). Thus, the robust stabilization problem reduces to the solution of the two Riccati equations simultaneously instead of $\gamma$-iteration process associated with traditional loop shaping such as mixed-sensitivity [1]. Two of $H_{\infty}$ loop shaping design procedures (LSDP) are available as shown in Figure (1); conventional loop shaping and target desired loop shaping.

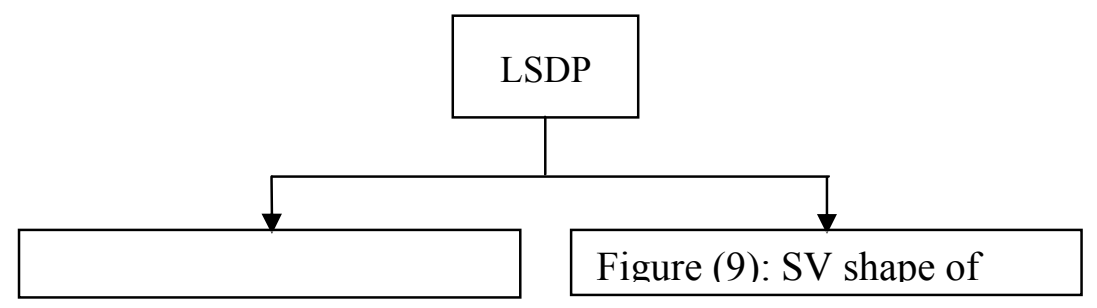

Figure (1): $H_{\infty}$ Loop Shaping Design Procedure

Here, the original open-loop system, $P$, is shaped by the two shaping functions, $W_{l}$ (pre-compensator) and $W_{2}$ (post-compensator) to match as closely as possible a desired shape to the singular values of the open-loop frequency response. The block diagram of this method is summarized in Figures (2) and (3).The shaped plant can be written as follows:

$$
P_{s}=W_{1} K_{\infty} W_{2}=:\left[\begin{array}{l|l}
A_{s} & B_{s} \\
\hline C_{s} & D_{s}
\end{array}\right]
$$




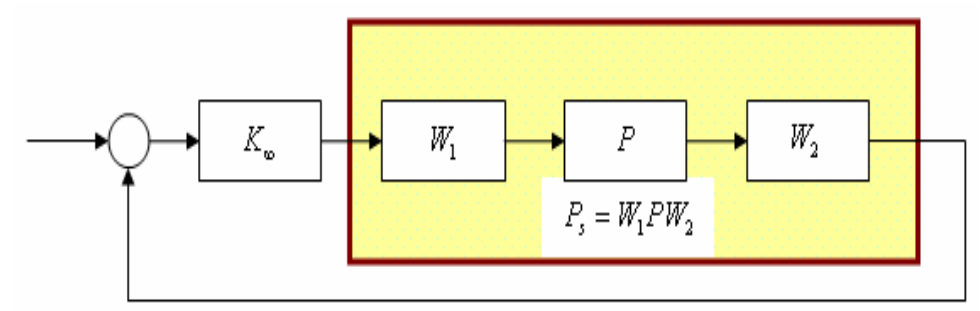

Figure (2): $P$ is shaped by $W_{1}$ and $W_{2}$

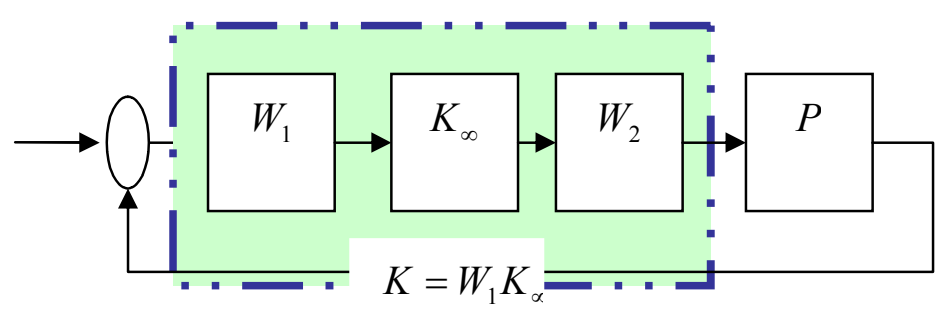

Figure (3): Final controller $K$ is constructed by combining $K_{\infty}$ with $W_{1}$ and $W_{2}$

Then, the controller is formed by combining the central controller $\mathrm{K}_{\infty}$ with the shaping functions $W_{1}$ and $W_{2}$ as shown in the Figure (3). The final controller plant can be written as follows:

$K=W_{1} P W_{2}=:\left[\begin{array}{l|l}A_{k} & B_{k} \\ \hline C_{k} & D_{k}\end{array}\right]$

\subsection{Target Desired Loop Shaping:}

This technique has been developed by Safonov-Le [2] for designing an optimal and stable minimum-phase Glover-McFarlane Pre-filter $W$ that locates (fitting) singular values of the open-loop frequency response plot to any desired singular values plot as precisely as possible. The algorithm combines a novel all-pass squaring-down compensator technique, of Safonov-Le, together with optimal Balanced Stochastic Truncation (BST) minimal realization techniques and normalized-coprime optimal $\mathrm{H}_{\infty}$ synthesis. Further, the Safonov-Le pre-filter has the important property that plant RHP zeros are left invariant; i.e., no performance-limiting RHP zeros and poles are introduced. The result is that the designer is completely relieved of task of manually computing the weight $W$. Designing an optimal loop shaping controller $K$ for plant $P$ with this algorithm is simple as specifying the desired loop shape ${ }_{d}$ [2]. The block diagram of this method is summarized in Figures (4) and (5). The shaped plant is 
written as:

$P_{s}=W P=:\left[\begin{array}{c|c}A_{s} & B_{s} \\ \hline C_{s} & D_{s}\end{array}\right]$

And the final robust controller is of the form:

$K=K_{\infty} W=:\left[\begin{array}{c|c}A_{k} & B_{k} \\ \hline C_{k} & D_{k}\end{array}\right]$

Where $W$ is the Safonov-Le pre-filter

In this paper, the $H_{\infty}$ optimization with target desired loop-shaping is selected for designing a robust controller to stabilize the longitudinal.

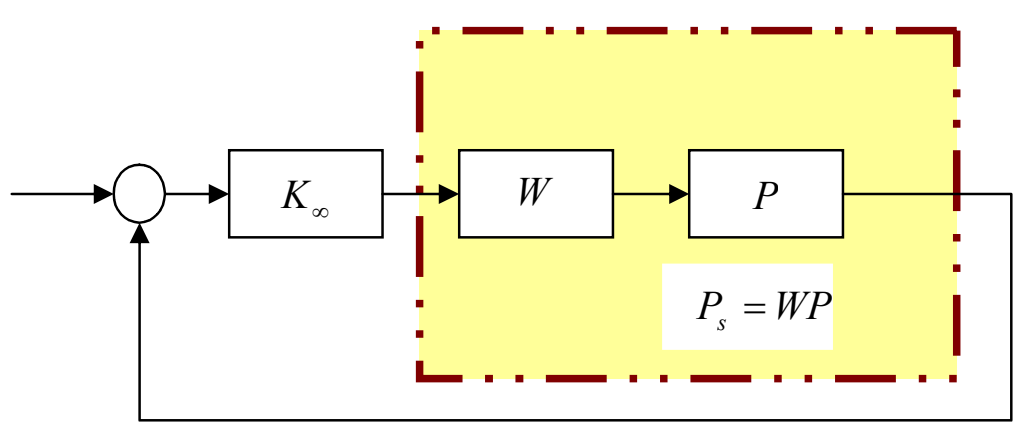

Figure (4): $P$ is shaped by $W$ and stabilized by $K_{\infty}$

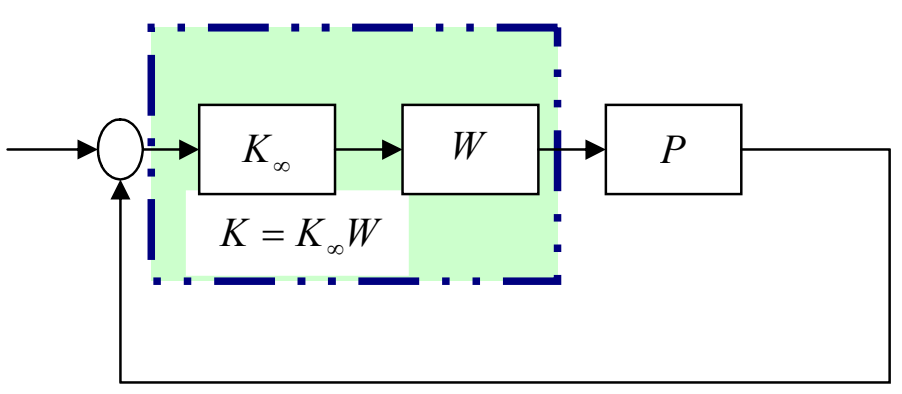

Figure (5): Final controller $K$ is constructed by combining $K_{\infty}$ with $W$

\section{Problem Formulation:}


Given a shaped plant $P_{s}$ and $A, B, C, D$ which represent the shaped plants in the state space form. The robust stabilization problem is to find a realizable and stablizable optimal robust controller $K$ such that

$\left\|\left[\begin{array}{c}K \\ I\end{array}\right](I-P K)^{-1} M^{-1}\right\|_{\infty} \leq \frac{1}{\varepsilon}=\gamma$

where $\gamma$ is the $\|\mathrm{H}\|_{\infty}$ from $\phi$, to $\left[\begin{array}{ll}\delta & y\end{array}\right]^{T}$,

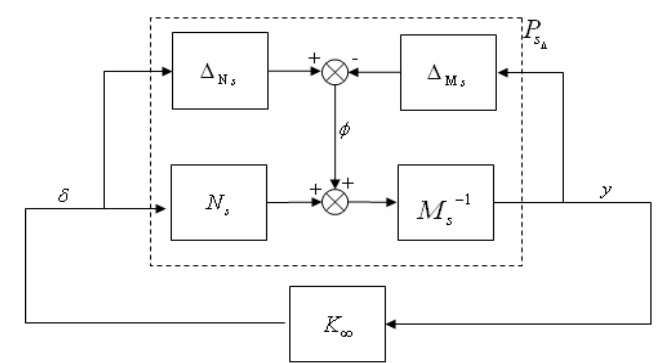

Figure (6): left coprime factor

Figure(6), and $(I-P K)^{-1}$ is the sensitivity function. Mc-Farlane and Glover have shown that, if the normalized coprime uncertainty is used, the optimal values of $\varepsilon_{\max }$ or $\gamma_{\text {min }}$ can be found directly without iteration from the following relation [3]:

$$
\gamma_{\text {min }}=\varepsilon_{\text {max }}{ }^{-1}=\left\{1-\left\|\left[\begin{array}{ll}
N_{s} & M_{s}
\end{array}\right]\right\|_{H_{\infty}}{ }^{2}{ }^{\frac{1}{2}}=(1+\rho(X Z))^{\frac{1}{2}}\right.
$$

Where: $\varepsilon$ is an uncertainty boundary, called stability margin, $\rho$ is the spectral radius (maximum eigenvalues), $Z$ and $X$ are the solutions to (GCARE) and (GFARE)

The Generalized Control Algebraic Riccati Equation (GCARE) and the Generalized Filter Algebraic Riccati Equation (GFARE) can be written as follows [1]:

$$
\begin{aligned}
& \left(A-B S^{-1} D^{T} C\right) Z+Z\left(A-B S^{-1} D^{T} C\right)^{T}-Z C^{T} R^{-1} C Z+B S^{-1} B^{T}=0 \\
& \left(A-B S^{-1} D^{T} C\right) X+X\left(A-B S^{-1} D^{T} C\right)^{T}-X B S^{-1} X+C^{T} R^{-1} C=0
\end{aligned}
$$

Where $S=I+D^{T} D \& \quad R=I+D D^{T}$

The controller which guarantees that:

$$
\left\|\left[\begin{array}{c}
K \\
I
\end{array}\right](I-P K)^{-1} M^{-1}\right\|_{\infty}<\gamma
$$

is given by

$$
X \approx\left[\begin{array}{c|c}
A+B F+\gamma^{2}\left(L^{T}\right)^{-1} Z C^{T}(C+D F) & \gamma^{2}\left(L^{T}\right)^{-1} Z C^{T} \\
\hline B^{T} X & -D^{T}
\end{array}\right]
$$

Where $F=-S^{-1}\left(D^{T} C+B^{T} X\right)$ \& $L=\left(1-\gamma^{2}\right) I+X Z$ and $(\mathrm{A}, \mathrm{B}, \mathrm{C}, \mathrm{D})$ are the minimum realization of $P_{s}$. Note that, if $\min ^{\gamma=\lambda_{\text {opt }}}, L$ becomes $L=\rho(X Z)+X Z$ [1].

\section{Controller Order Reduction:}


Due to the high-order controllers, the robust control system design necessitates the reduction of the control system order. It is well-known that advanced control theories produce high order controllers compared to classical techniques. The order can be reduced using several ways as depicted in Figure (7) [4]. In this paper, a high order controller is designed first stage and then its order is reduced in the second stage because there is freedom in choosing the final order of the controller. The main approaches which are available to reduce are [4]:

1. Balanced truncation

2. Balanced residualization.

3. Optimal Hankel norm approximation

Balanced truncation and balanced residualization approaches are convenient for removing the high frequency or fast modes of a state space realization [4], while an optimal Hankel norm approximation approach is used

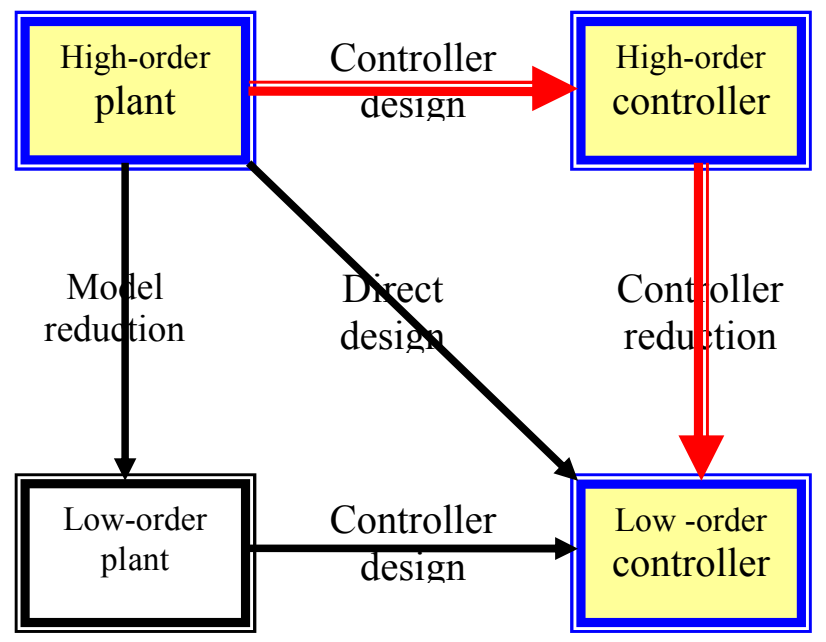

Figure (7): Design flow of low-order controllers to remove the unobservable and/or uncontrollable modes [3]. Here the optimal Hankel norm approximation approach is selected to bound the additive error. The Hankel singular values, named after Hermann Hankel, provide a measure of energy for each state in the system. They are the basis for balanced model reduction, in which high energy states are retained while low energy states are discarded. The reduced model retains the important features of the original model [5]. The pitch-rate SAS inner loop, which is designed to increase the damping of the short-period oscillation. This can be accomplished by adding an inner feedback loop utilizing a rate gyro for sensing the pitch-rate controlled variable. The CAS outer loop consists of the feedback of the output $Q$. The error difference between $Q_{\text {com }}$ and $Q$ is fed to a PID controller to improve tracking performance. Since there is a sign change present inherently in the aircraft dynamics associated with the relationship of the pitch-rate to elevator deflection, the feedback signal is added to the SAS command voltage signal.

\section{Longitudinal dynamics control design:}

Before starting the design procedure, the open loop frequency response of the linear model is analyzed in some details with concentration on its shape in the low and high frequency regions. The significance of this analysis is to show the actuators (elevator) input effect on all outputs states individually [1]. The information gain from this analysis can be used to aid the design of controller. 


\subsection{Open-Loop Singular Values:}

The uncompensated longitudinal channel loop is summarised in the block diagram of Figure (8) where the plant dynamic model is obtained from the previews works [6]. The output matrix is set to extract the longitudinal states individually as shown bellow:

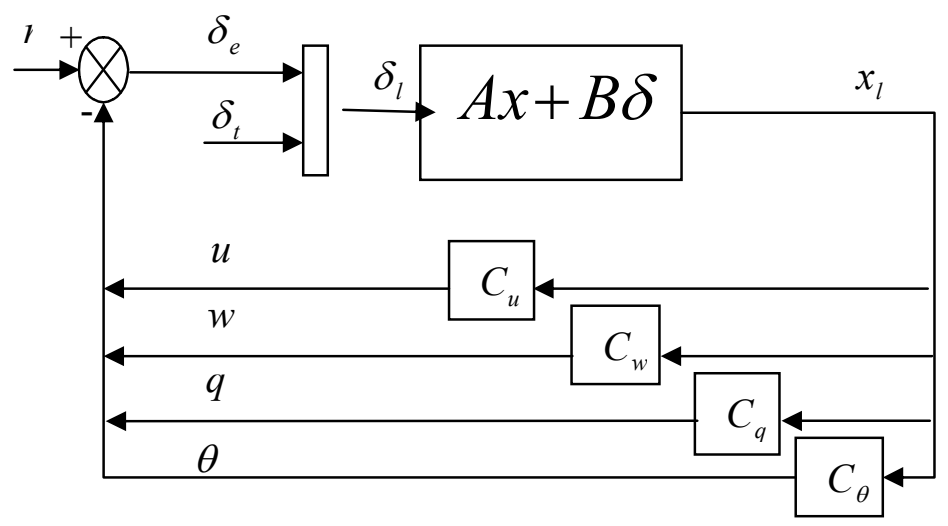

Figure (8): Block diagram of uncompensated longitudinal plant

$C_{u}=\left[\begin{array}{llll}1 & 0 & 0 & 0\end{array}\right]$ is the axial velocity

state $[\mathrm{m} / \mathrm{s}], C_{w}=\left[\begin{array}{llll}0 & 1 & 0 & 0\end{array}\right]$ is the normal velocity $[\mathrm{m} / \mathrm{s}], C_{q}=\left[\begin{array}{llll}0 & 0 & 1 & 0\end{array}\right]$ is the pitch rate state $[\mathrm{rad} / \mathrm{s}]$, $C_{\theta}=\left[\begin{array}{llll}0 & 0 & 0 & 1\end{array}\right]$ is the pitch angle state [deg]. The of singular values for the uncompensated longitudinal plant from the outputs states individually to the elevator actuator is shown in Figure (9)

the following observations can be obtained Figure (9):

1. For the pitch angle state to elevator input

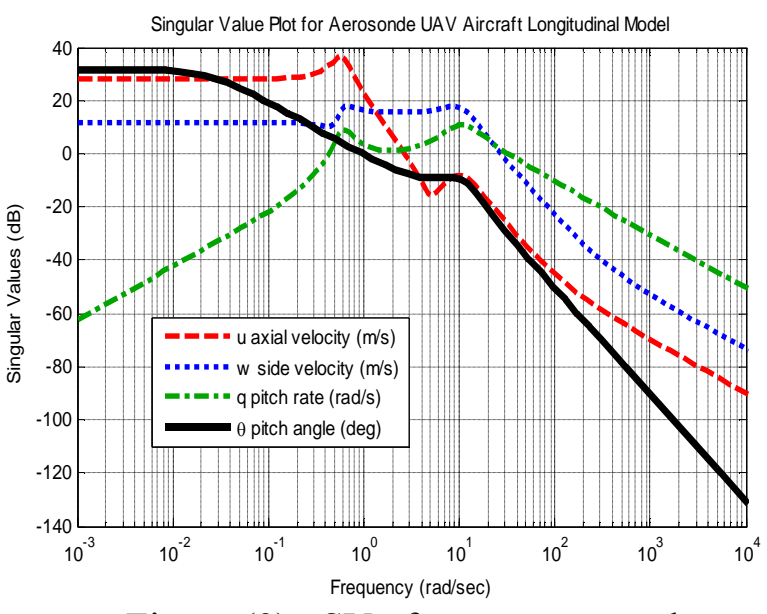

Figure (9): SV of uncompensated longitudinal plant

state

loop, it is expected to shave highest gain in the low frequency region and steeply roll-off (lowest gain) in the high frequency region for good disturbance rejection and noise attenuation.

2. For the axial velocity state to elevator input loop, it is expected to have smaller gain than the pitch angle state in the low frequency region and low gain but greater than the pitch angle state loop in the high frequency region.

3. For the normal velocity state to elevator input loop, it is expected to have smaller gain than the axial velocity loop in the low frequency region and larger gain than the axial velocity loop in the high frequency region.

4. For the pitch rate state to elevator input loop, it is expected to have smallest gain in the low 
frequency region and largest gain in the high frequency region.

It is clear that the pitch angle state guarantees the performance and robustness requirements better than the other states since its singular value shape revealed that it has highest gain in the low frequency region and lowest gain in the high frequency region relative to the singular values shapes associated to the other states.

\subsection{Target Desired Loop Shape Selection:}

The target desired loop shape represents the design specifications in the frequency domain. The determination of the target desired loop shape is an iterative process. The target desired loop shape found to realize the specifications has the following transfer function [6]:

$$
G d=\frac{(2.8 s+0.3)}{(s+0.05)^{2}}
$$

The singular value of the desired loop shape is shown in Figure (10). The accuracy with which the control design matches the target desired loop is depicted with the dotted lines around the desired loop shape.

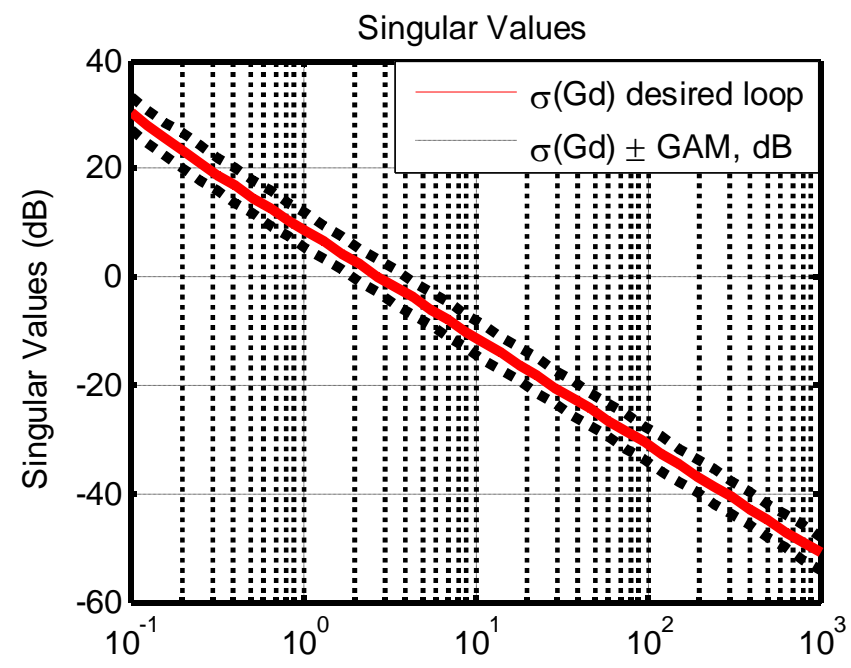

Figure (10): SV shape of target desired

\subsection{Robust Controller:}

The linear time invariant robust controller is found by shaping the central controller with shaping function. The state space form of the robust controller is obtained as:

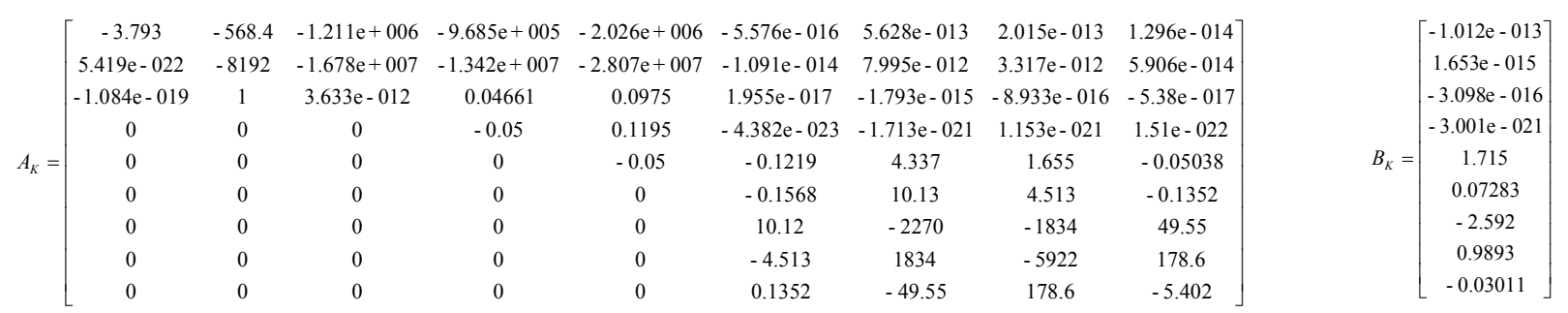




$$
D_{K}=[1.137 \mathrm{e}-013]
$$

$C_{K}=[0.1519-274.6$

The designed robust controller is optimally shaped and fitted the open frequency response of the plant to match closely as possible a desired loop shape shown in Figure (11). The slope of the loop shape is increased at low frequency as to reduce the high frequency gain for noise attenuation.

The sensitivity function $S$ and complementary sensitivity function $T$ of closed loop system with $K$ is shown in (12). It is clear that design requirements disturbance rejection and noise attenuation are satisfied. The LSDP controller $K$ ensures a stability margin $\varepsilon=0.6957$ or $\gamma=1.4375$. This is a good with respect to the robust stability, a design is usually considered successful $\varepsilon \geq 0.25$ or $\gamma \leq 4$ [1].

The frequency response of system is the figure (13). The gain margin is from $\infty$ into $65[\mathrm{~dB}]$, the phase margin from -81.2107 into $88.6861[\mathrm{deg}]$, and bandwidth is modified from 0.9677 into The modified controller order is reduced order system, equation (15), to a thirdby using Hankel norm approximation Hankel singular value plot of the LSDP shown in Figure (14). It showed that the

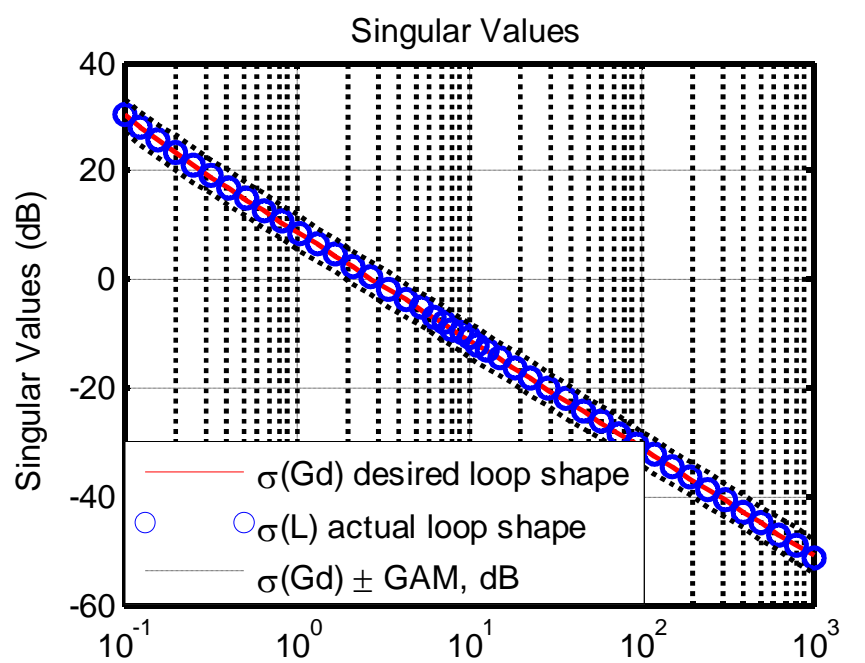

Figure (11): Fitting open-loop SV of pitch channel into desired loop shape

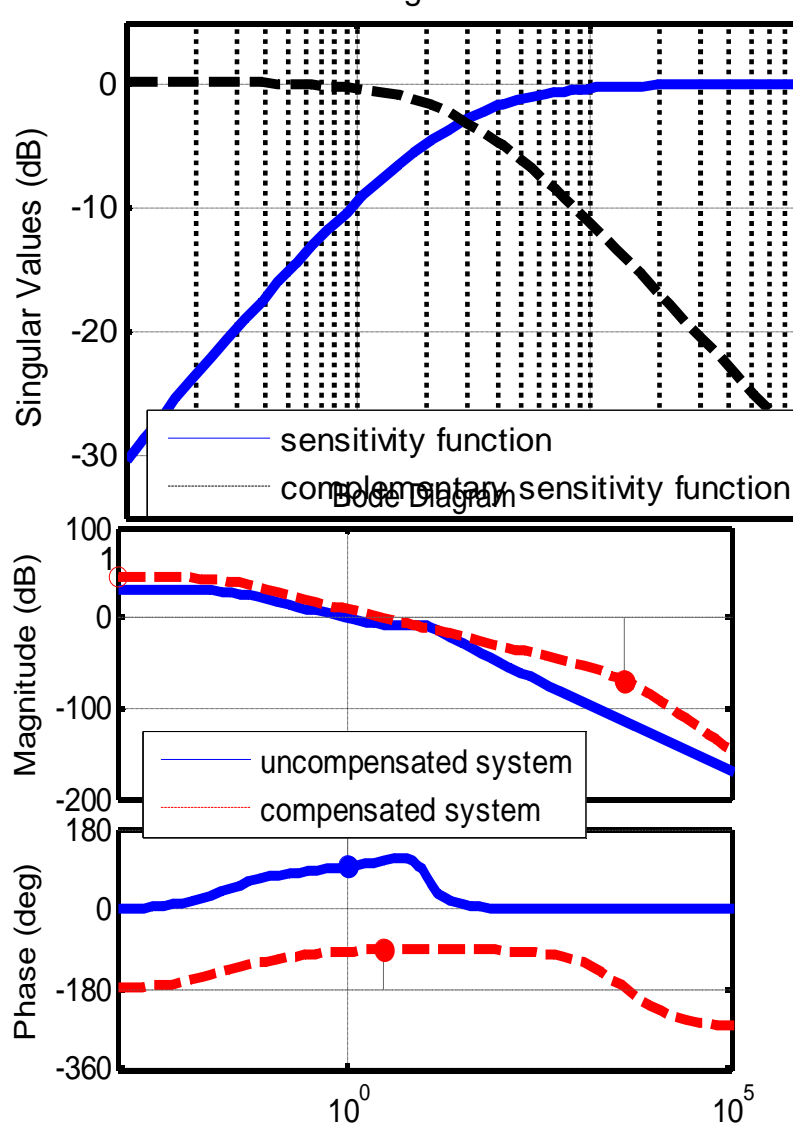
Figure (13): Bode diagram of compensated controller $K$ and uncompensated pitch svstem loop

$G_{d} \quad$ as

plant

range so

good

the

Figure

for the

level

because

if

shown in modified modified the $2.8637[\mathrm{rad} / \mathrm{s}]$. from ninthorder system technique. The controller is 
has most of its energy stored in states 1 through 3. It is expected that these states preserve most of the dynamic response of the system.

Starting from the first-order, the singular values shape $\mathrm{s}$ are compared singular value shape of the full-order shown in Figure (15). This figure following observations:

- The first-order controller response is the full-order response up to about $[\mathrm{rad} / \mathrm{s}]$.

- The second-order controller response is the full-order response up to about 60

- The third-order controller response is

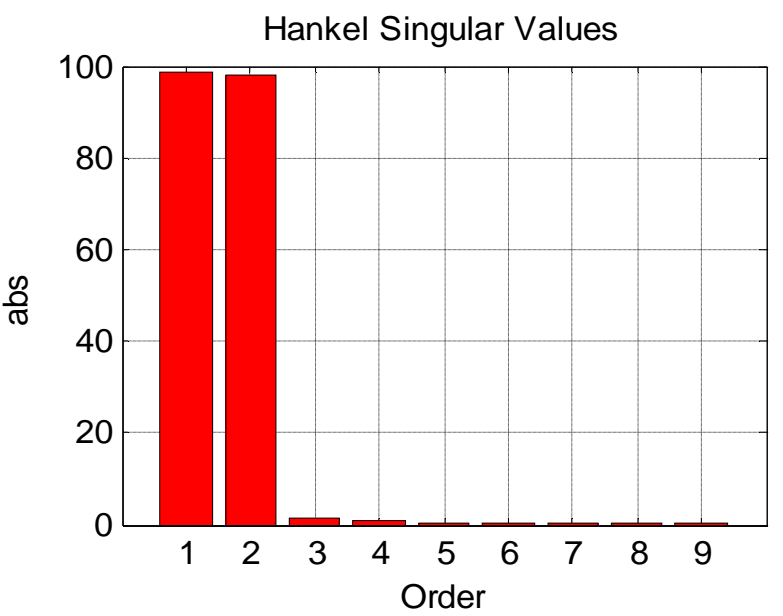

Figure (14): Bar graph of Hankel SV of LSDP controller for pitch channel

controller's with the controller as clarifies the

not close to 2950

not close to $[\mathrm{rad} / \mathrm{s}]$. close to the full-order controller at all.

Therefore, the controller is reduced to the third-order, for which the state space form is as follows:

$$
\begin{aligned}
& A_{K r_{p}}=\left[\begin{array}{ccc}
-8198 & -4102 & -38 \\
4102 & -0.003417 & -0.02528 \\
-38 & 0.02608 & -3.215
\end{array}\right] \\
& B_{K r_{p}}=\left[\begin{array}{c}
-1271 \\
0.8183 \\
-2.987
\end{array}\right] \\
& C_{K r_{P}}=\left[\begin{array}{lll}
1271 & 0.8194 & 2.987
\end{array}\right] \\
& D_{K r_{P}}=[1.137 \mathrm{e}-013]
\end{aligned}
$$

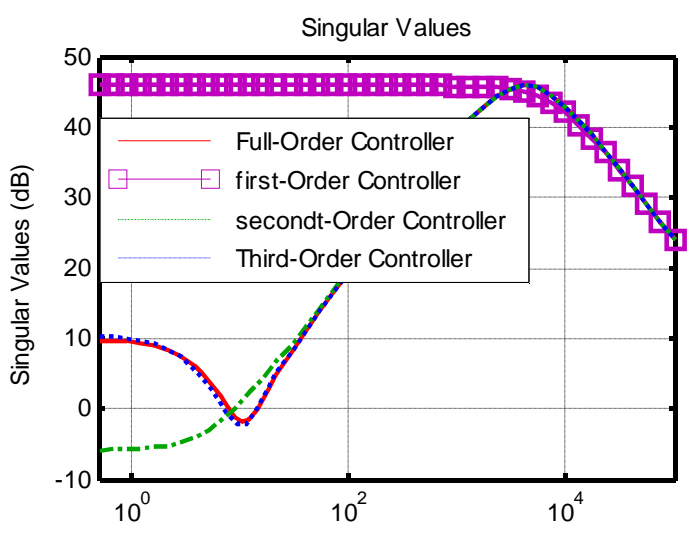

Figure (15): SV shape of full- and reduced order controllers for pitch

The optimal solution is given by:

$$
K=R_{w}^{-1} B^{T} P
$$

where $P=P^{T} \geq 0$ is the unique positive semi-definite solution of the algebraic Riccati amuation. 


\section{Controllers Comparison:}

The robust (SDOF) controller is compared with classical (SDOF \& TDOF) controllers that are designed in previews work which can be found in [6] and [7]. In comparison with the classical PID design, the robust LSDP controller revealed superior robust stability with equivalent level of nominal performance as shown in the following figures: 


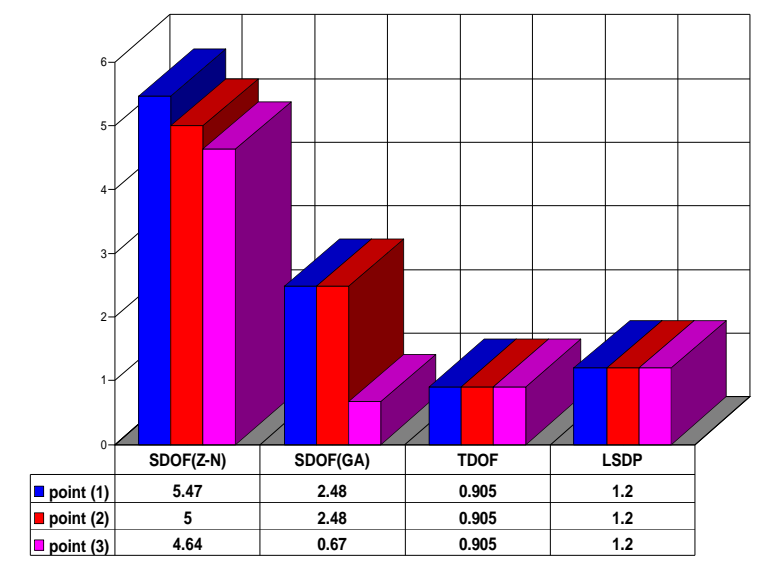

Figure (16): Settling Time (sec)

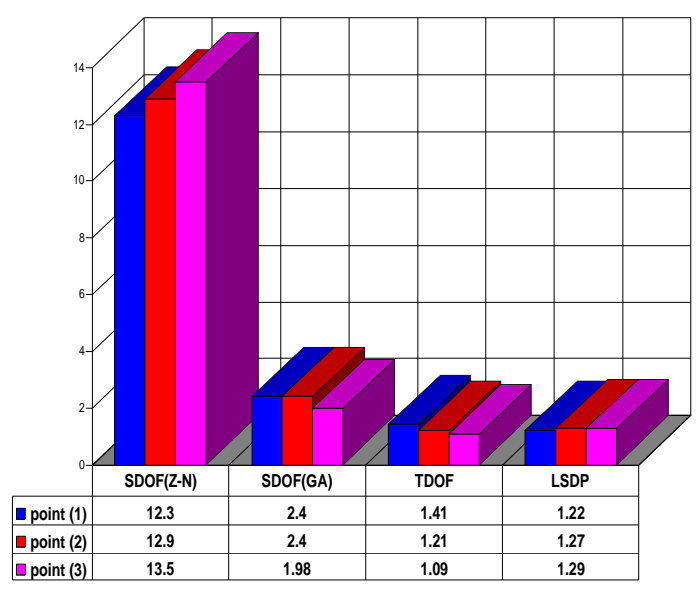

Figure (18): Maximum Overshoot
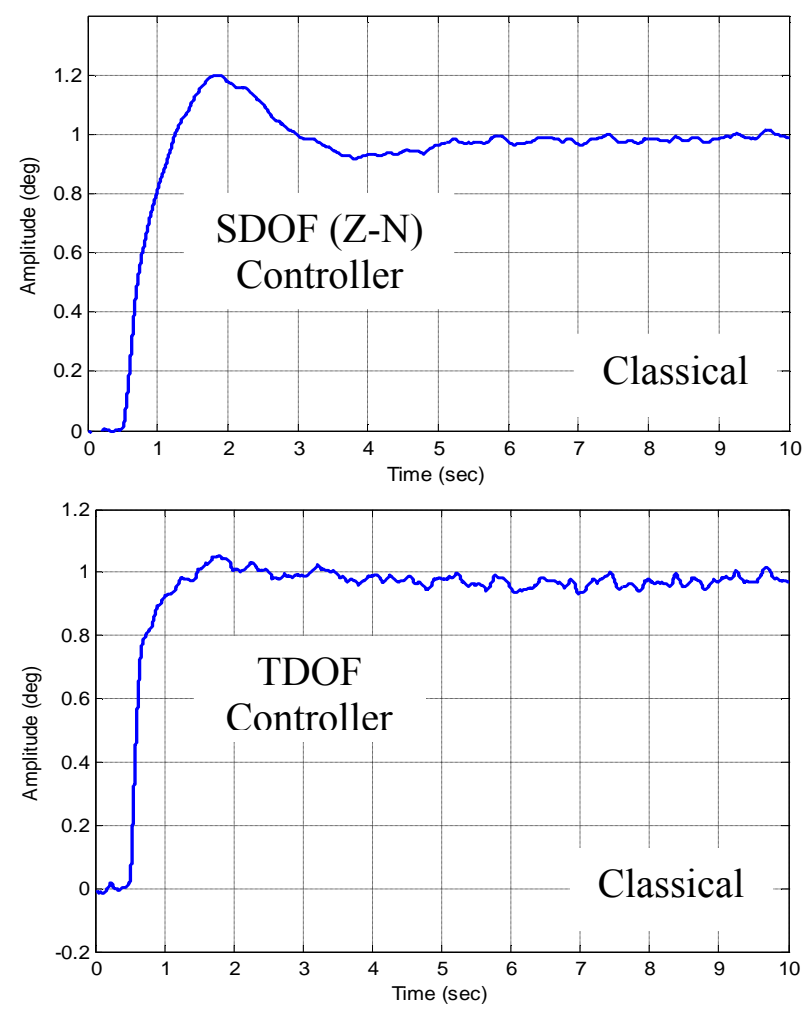

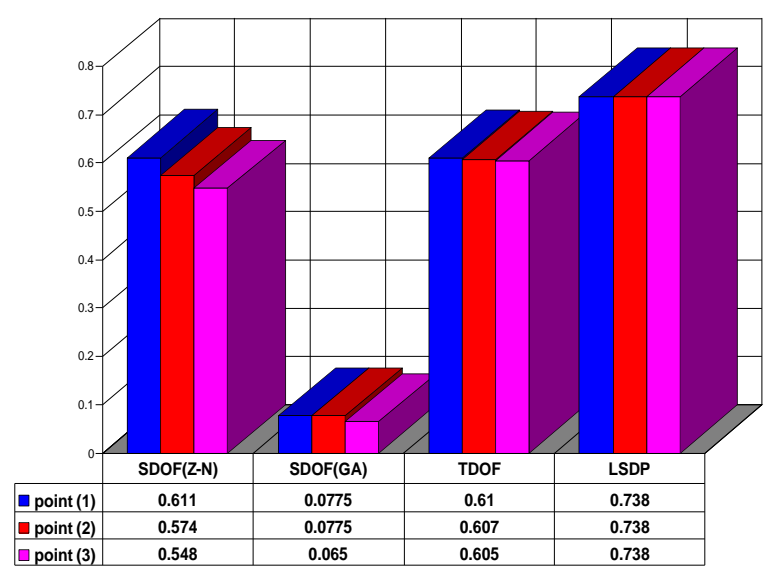

Figure (17): Rise Time (sec)

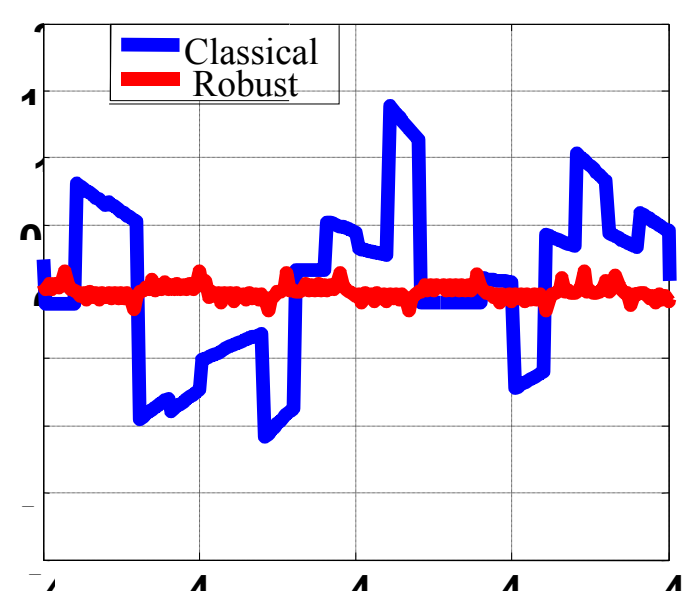

Figure (19): Control Signal due to white noise and reference
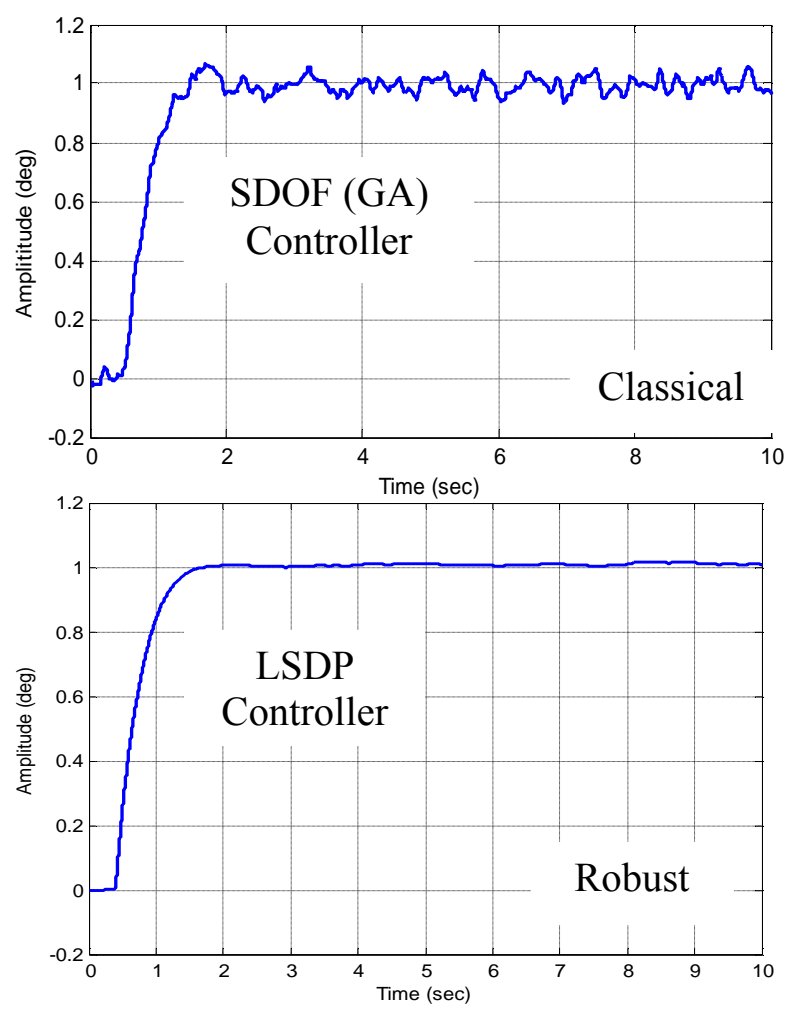


\section{Conclusions:}

The design of robust controllers for longitudinal dynamics is presented. The analysis of aircraft open-loop singular values is considered in addition to the $\mathrm{H}_{\infty}$ loop shaping design procedure (LSDP). Then, the controller order reduction is performed. A maximum evaluation is successfully made in linear simulation for singular values as an ultimate test of the final reduced-order controller. The robust controller is then compared with the classical ones in terms of disturbance rejection, noise attenuation, unmodeled dynamics, and control effort.

\section{References:}

[1] Salah I. AlSwailem. Application of Robust Control in Unmanned Vehicle Flight Control System Design, Ph.D Thesis, Cranfiled, England, 2003-2004

[2] G.J. Balas, A.K. Packard, M.G. Safonov and R.Y. Chiang, Next generation of tools for robust control, Proc. American Control Conf., Boston MA, June 30 - July 2, 2004

[3] Da-Wei Gu, Petko H. Petkov and Mihail M.Konstantinov, Robust Control Design with MATLAB, Springer

[4] Sigurd Skogestad and Ian Poastlewaite. Multivariable Feedback Control Analysis and Design, Second Edition, Wily, 2005

[5] Robust Control Toolbox Version 2

[6] Capt. Eng. Elfatih G. Hamdi, 'UAV Robust Flight Control Systems' M.Sc Thesis, MTC, Cairo, 2008

[7] Elfatih G. Hamdi, Ahmed M. Youssef, and Gamal A. Elsheikh, $6^{\text {th }}$ International Conference on Electrical Engineering, MTC, Cairo, 27-29 May 2008

\section{Nomenclatures:}

A, B, C, D matrices used in the state space description

SDOF single degree of freedom

TDOF two degree of freedom

Z-N Ziegler Nichol

GA genetic algorithm

LSDP loop shaping design procedure 